vertreten wurde, einen Rückgriff auf $₫ 267$ StGB zuzulassen und die Rechtsfolgen stets allein den $\$ \$ 277$ Var. 2, 3, 279 StGB zu entnehmen ${ }^{16}$, mochte das zwar die unbilligen Ergebnisse vermeiden. Doch kam man nicht umhin, darin eine Hilfskonstruktion zu erblicken, die sich um die notgedrungene Folgenbeseitigung einer defizitären Rechtslage bemühte. Vorzugswürdig erschien es aber, dem dazu berufenen Gesetzgeber die Anpassung der Rechtslage zu überlassen. Dies ist nun durch Gesetz v. 22.11.2021 (BGBl. I S 4906 ff.) geschehen (dazu unter 7.).

6. Neben möglichen Strafbarkeiten im Strafgesetzbuch hat das Gericht auch Strafbarkeiten aus dem Infektionsschutzgesetz erwogen. Hier hatte der Gesetzgeber im Juni diesen Jahres neue Strafvorschriften eingeführt ( $\$ S 74$ Abs. 2, 75a IfSG), die dem Kampf gegen Impfausweisfälschungen dienen sollten. Auch diese Vorschriften sah das LG Osnabrück zutreffend als nicht erfüllt an. Dabei wird die komplizierte Verweiskette von der Strafvorschrift des $\$ 74$ Abs. 2 hin zu $\$ 22$ Abs. 1 IfSG übersichtlich aufgeschlüsselt. Diese führt letztlich dazu, dass ein Handeln entgegen $\$ 22$ Abs. 1 IfSG eine notwendige Voraussetzung für eine Tatbegehung nach $\$ 74$ Abs. 2 IfSG ist. Zutreffend hat das Gericht erkannt, dass gegen $\$ 22$ Abs. 1 IfSG nur handeln kann, wer dem dort genannten Kreis der „,impfberechtigten Person“ unterfällt. In der aktuellen Praxis sind dies Ärzte, wenngleich auch die Möglichkeiten von Schutzimpfungen durch Apotheker. Infolgedessen wird $\$ 74$ Abs. 2 IfSG zu einem Sonderdelikt, das nur von Ärzten und Apothekern erfüllt werden kann. Das Fälschen durch andere Personengruppen, bspw. professionelle Fälscher, wird damit nicht vom Tatbestand erfasst. Das hat auch Folgen für eine - hier in Betracht kommende - Strafbarkeit nach $\$ 75$ a Abs. 2 Nr. 1 IfSG, die den Gebrauch von Impfausweisen in den Blick nimmt. Voraussetzung nach dieser Vorschrift ist nämlich der Gebrauch einer in $\$ 74$ Abs. 2 IfSG bezeichneten nicht richtigen Dokumentation. Eine Strafbarkeit hiernach kommt im Ergebnis damit richtigerweise ebenfalls nur in Betracht, wenn die Impfung in dem gebrauchten Ausweis von einem Arzt eingetragen wurde ${ }^{17}$. Fälschlicherweise hat das Gericht im Zuge dessen \75a Abs. 2 Nr. 1 IfSG selbst zum Sonderdelikt erklärt. Bei diesem handelt es sich jedoch um ein Allgemeindelikt. Lediglich mit Blick auf die Herstellung des tauglichen Tatmittels müssen - wie gezeigt - die Voraussetzungen des Sonderdelikts des $\$ 74$ Abs. 2 IfSG vorliegen. Im Ergebnis scheidet eine Strafbarkeit nach $\$ 75$ a Abs. 2 Nr. 1 IfSG im hiesigen Fall dennoch aus. Schließlich wurde kein Ausweis in der Apotheke vorgelegt, in welchem die Impfung tatsächlich durch einen Arzt unrichtig eingetragen worden ist. Der Fehler des Gerichts war damit folgenlos.

7. Abschließend bleibt festzuhalten, dass die Auslegung des LG Osnabrück die vormals defizitäre Rechtslage in den entscheidenden Punkten zutreffend offengelegt hat. Dadurch hat das Gericht den politischen Diskurs in diesem Problembereich weiter vorangetrieben. Es ist nachdrücklich zu begrüßen, dass auch die $\$ \$ 277,279$ StGB a. F. im Zuge der großen Reform des Infektionsschutzrechts durch Gesetz v. 22.11.2021 (BGB1. I S. 4906 ff.) umgestaltet wurden. Der Gesetzgeber hat u.a. \$277 Var. 2., 3 StGB a.F. gestrichen, die Vorschrift also auf den Wahrheitsschutz reduziert und damit den sich mit der ,allgemeinen“ Urkundenfälschung überschneidenden Echtheitsschutz aus der Vorschrift verbannt. Durch den in $\$ 279$ StGB enthaltenen Verweis auf $\$ 277$ StGB zeitigt dies auch Wirkung für die Fälle reinen Gebrauchens gefälschter Impfausweise. Das Problem der unbilligen, aber bislang hinzunehmenden Sperrwirkung der \$S 277, 279 StGB gegenüber der , allgemeinen“ Urkundenfälschung aus $\$ 267$ StGB ist damit gelöst. Für Verfahren, in denen die Tatbegehung noch vor Inkrafttreten der Reform erfolgte, sind jedoch weiterhin Ergebnisse wie im Beschluss des LG Osnabrück zu erwarten. Denn stuft man - wie hier vertreten - die alte gegenüber der neuen Rechtslage als milder ein, findet der lex-mitior-Grundsatz aus $\$ 2$ Abs. 3 StGB Anwendung. Die Konsequenz: In diesen Fällen ist zu Gunsten der Täter die für sie günstigere alte Rechtslage anzuwenden, sodass die Sperrwirkung wohl noch in einigen Verfahren zur Straflosigkeit führen wird.

16) Dafür Fischer, StGB, 67. Aufl. 2020, $\$ 277$, Rdnr. 11

17) A.A. Krüger/Sy, GesR 2021, 626, 632f., die meinen, der Wortlaut ließe auch die Bestrafung wegen des Gebrauchs eines durch Privatpersonen gefälschten Impfausweises zu. Wenn sie dazu erklären, in $\$ 75$ a Abs. $2 \mathrm{Nr}$. 1 IfSG sei immerhin nicht die Rede davon, der später gebrauchte Impfausweis müsse aus einer Tat gemäß $\$ 74$ Abs. 2 IfSG erlangt worden sein, ist das zwar zutreffend. Doch blenden sie aus, dass ein Verweis auf diese Vorschrift keinen Sinn ergibt, wenn man sie nicht in hier vertretenen Art versteht. Hätte der Gesetzgeber in $\$ 75$ a Abs. 2 Nr. 1 IfSG ,unrichtige Dokumentationen der Schutzimpfung gegen das Coronavirus SARSCoV-2“ gemeint, hätte er dies schlichtweg schreiben können. Ein Verweis auf nur einen Teil einer - ohnehin gerade erst und gemeinsam mit $\$ 75$ a IfSG eingeführten - anderen Strafvorschrift wäre nicht notwendig und nur irreführend gewesen. Letztlich hat wohl aber auch der Gesetzgeber allein den Gebrauch von durch Ärzte unrichtig ausgestellten Impfausweisen als von $\$ 75$ a Abs. 2 Nr. 1 IfSG erfasst angesehen. Hinsichtlich der neuen Strafvorschriften stellte dieser fest, dass Konkurrenzen zu den $\$ \$ 278,279$ IfSG auftreten können (BT-Dr. 19/29870, S. 34). Wenn aber z. B. ein Nichtarzt einen Impfausweis fälscht und diesen gegenüber einer Behörde anwendet, ist $\$ 277$ StGB erfüllt. Nach Lesart von Krüger/Sy müsste zugleich $\$ 75$ a Abs. 2 Nr. 1 IfSG einschlägig sein. Der Gesetzgeber hat eine Konkurrenz zwischen den beiden Vorschriften aber zu Recht ausdrücklich nicht in Betracht gezogen.

https://doi.org/10.1007/s00350-021-6084-7

\section{Teilnahme am Regelunterricht (Präsenzunterricht) in der Schule ohne Mund-Nasen-Bedeckung}

GG Art. 2 Abs. 1 u. 2, 19 Abs. 4; VwGO §§80 Abs. 5,123 Abs. 1 S. 2; ZPO §§920 Abs. 2 ZPO, 294; IfSG §§32 S. 1 u. 2, 28a Abs. 1 S. 1; Nds. Corona-Verordnung §§3 Abs. 6, 13 Abs. 1 S. 4 ff.; NSchG §31 Abs. 1, Abs. 10 Nr. 1 lit. h).

1. Die Verpflichtung zum Tragen einer Mund-NasenBedeckung stellt grundsätzlich - auch in der Schule eine zulässige Schutzmaßnahme dar (vgl. Niedersächsisches OVG, Beschl. v. 14.8.2020 - 13 MN 300/20).

2. Eine Ausnahme von der Maskenpflicht kann bei medizinischer Indikation bestehen, in niedersächsischen Schulen entweder nach $₫ 3$ Abs. 6 CoronaVO oder $\$ 13$ Abs. 5 CoronaVO i. V. mit dem Rahmen-Hygieneplan Corona Schule.

3. Für den Wegfall der Verpflichtung bedarf es der Glaubhaftmachung, die die Vorlage eines für die Verwaltung prüffähigen Attests erfordert, da dieses einen rechtlichen Vorteil vermittelt.

4. An das vorzulegende (aktuelle) Attest sind inhaltliche Anforderungen dergestalt zu stellen, dass die konkret aufgrund des Tragens einer Maske im Unterricht alsbald zu erwartenden gesundheitlichen Beeinträchtigungen nachvollziehbar benannt werden unter Darlegung woraus diese im Einzelnen resultieren; und zwar unter Nennung etwaiger Vorerkrankungen sowie der für die Einschätzung maßgeblichen Grundlage.

VG Braunschweig, Beschl.v. 19. 11.2020 - 4 B 397/20

Eingesandt vom Vorsitzenden Richter

am VG Braunschweig Claus Brölsch, Braunschweig;

bearbeitet von Rechtsanwalt Benedikt Nethe,

CausaConcilio Koch \& Partner mbB Rechtsanwälte,

Bei den Gerichten - Deliusstraße 16, 24114 Kiel, Deutschland 
Problemstellung: Kernfrage der Entscheidung ist, ob Schüler vom Tragen einer Mund-Nasen-Bedeckung befreit sind, wenn sie ein Attest vorlegen können, welches ihnen pauschal attestiert, dass ihnen das Tragen einer Maske aus ärztlicher Sicht nicht empfohlen sei. Die Entscheidung ist dabei in den Kontext der Coronapandemie eingebettet und nimmt zwar speziell die Niedersächsische Corona-Verordnung in den Blick, ist aber mit graduellen Anpassungen auf das gesamte Bundesgebiet übertragbar. Seit Beginn der Pandemie wurden regelmäßig neue Maßnahmen zur Bekämpfung gegen das Pandemiegeschehen eingeführt. Diese waren ebenso regelmäßig Gegenstand gerichtlicher Entscheidungen. Erneut waren die grundrechtlichen Positionen, also das Interesse des Einzelnen, geschützt durch das Grundrecht auf allgemeine Handlungsfreiheit, gegen den Gesundheitsschutz vom Gericht gegeneinander abzuwägen. In stringenter Fortführung der Rechtsprechung, dass die Verpflichtung zum Tragen einer Mund-Nasen-Bedeckung grundsätzlich auch in der Schule eine zulässige und verhältnismäßige Schutzmaßnahme darstellt, stand nunmehr die Überprüfung der Ausnahmevorschriften auf Befreiung von dieser Verpflichtung an. In dem vom VG Braunschweig entschiedenen Fall hatten die gesetzlichen Vertreter der antragstellenden Schüler ein ärztliches Attest vorgelegt, aus dem (lediglich) hervorging, dass sich die ASt. in der hausärztlichen Gemeinschaftspraxis in ambulanter Behandlung befinden und aus ärztlicher Sicht nicht empfohlen sei, eine Maske zu tragen.

Benedikt Nethe

Zum Sachverhalt: Die ASt. begehren im Wege der einstweiligen Anordnung die Feststellung, dass sie ohne das Tragen einer MundNasen-Bedeckung am Regelunterricht teilnehmen dürfen sowie die Feststellung, dass sie nicht verpflichtet sind, mitzuteilen, welche konkret zu benennende gesundheitliche Beeinträchtigung aufgrund des Tragens der Mund-Nasen-Bedeckung im Unterricht alsbald zu erwarten ist und woraus diese im Einzelnen resultiert oder aber relevante Vorerkrankungen konkret zu benennen.

Die am H. und am I. geborenen ASt. wurden am J. 2020 am F. in A-Stadt aufgenommen. Für beide ASt. legten die gesetzlichen Vertreter ein ärztliches Attest der Hausärztlichen Gemeinschaftsmaxis K. aus B-Stadt v. 25.6.2020 vor. Daraus geht hervor, dass sich die ASt. dort in ambulanter Behandlung befinden und aus ärztlicher Sicht nicht empfohlen sei, eine Maske zu tragen. Weitere Angaben sind nicht enthalten.

$[\ldots]$

Mit Schreiben v. 2.11.2020 wies die Schule auf die geltende Corona-Verordnung hin und bat um Vorlage eines entsprechenden Attestes bis zum 9.11.2020 in der Schule, das den in dem Schreiben genannten Voraussetzungen entspricht. Mit E-Mail v. 2.11.2020 wiesen die ASt. diese Forderung zurück. [...]

Mit Schreiben v. 9.11.2020 teilte der Prozessbevollmächtigte der ASt. der Schule mit, er halte die Forderung, eine Mund-NasenBedeckung zu tragen beziehungsweise, da ein Befreiungstatbestand geltend gemacht werde, anzugeben, welche konkret zu benennende gesundheitliche Beeinträchtigung auf Grund des Tragens der Mund-Nasen-Bedeckung im Unterricht zu erwarten sei und die Bekanntgabe der entsprechenden Grundlage der Einschätzung, für rechtswidrig, da sie gegen die grundrechtlich geschützten Persönlichkeitsrechte verstoße. Die ASt. seien nicht verpflichtet, den Hintergrund der Erkrankung, die zur Befreiung vom Mund-Nasen-Schutz führe, bekanntzugeben. Er forderte die Schule unter Fristsetzung bis zum 10.11. 12.00 Uhr auf, mitzuteilen, dass die ASt. aufgrund der vorgelegten Atteste von der Verpflichtung zum Tragen eines MundNasen-Schutzes in der Schule befreit seien.

Die ASt. haben am 13.11.2020 einen Antrag auf Gewährung vorläufigen Rechtsschutzes gestellt.

Diesen begründen sie im Wesentlichen damit, sie seien nicht verpflichtet, den Grund ihrer Erkrankung mitzuteilen oder behandelnde Ärzte von ihrer Schweigepflicht zu entbinden, so dass diese den Grund der Erkrankung mitteilen. Dies ergebe sich aus dem allgemeinen Persönlichkeitsrecht der ASt. sowie aus datenschutzrechtlichen Erwägun- gen. [...] Sie hätten ein ärztliches Attest vorgelegt, aus dem sich ergebe, dass sie aus gesundheitlichen Gründen nicht zum Tragen einer MundNasen-Bedeckung geeignet seien. Weitere Informationen müssten sie nicht liefern. Sie gingen derzeit davon aus, dass sie ohne Mund-NasenSchutz nicht am Regelunterricht teilnehmen dürften und seien daher nicht mehr zum Unterricht erschienen. Da ihnen hierdurch allerdings erhebliche Nachteile bei der Bewältigung des Lernstoffes drohten, sei der Erlass einer einstweiligen Anordnung geboten.

$[\ldots]$

Eine Befreiung von der Pflicht zum Tragen einer Mund-NasenBedeckung stehe bereits dem Wortlaut nach nicht zur Disposition des AG. Nach $₫ 3$ Abs. 6 der Nds. Verordnung über Maßnahmen zur Eindämmung des Corona-Virus SARS-CoV-2 (Nds. CoronaVerordnung) sei eine Befreiung durch den Ag. nicht vorgesehen.

Unabhängig davon hätten die ASt. nicht glaubhaft gemacht, dass sie von der Pflicht zum Tragen einer Mund-Nasen-Bedeckung auf dem gesamten Schulgelände und insbesondere während des Unterrichts aus gesundheitlichen Gründen durch ärztliches Attest befreit seien. Neben den Bestimmungen in der CoronaVO ergebe sich eine Pflicht zum Trage einer Mund-Nasen-Bedeckung auch aus der Allgemeinverfügung zum Schutz der Bevölkerung vor der Verbreitung des Corona-Virus SARS-CoV-2 auf dem Gebiet des Landkreises AStadt v. 7.11.2020.

Gemäß $₫ 3$ Abs. 6 CoronaVO seien bestimmte Personen unter bestimmten Voraussetzungen von der Verpflichtung zum Tragen einer Mund-Nasen-Bedeckung ausgenommen. Die inhaltlichen Anforderungen an ein ärztliches Attest beziehungsweise eine vergleichbare amtliche Bescheinigung seien durch die Rundverfügung Nr. 26/2020 der Nds. Landesschulbehörde v. 30.10.2020 (zur Anwendung der Nds. Verordnung über Maßnahmen zur Eindämmung des Corona-Virus SARS-CoV-2) konkretisiert worden. Um der Schule eine sachgemäße Prüfung der Befreiung von der sogenannten Maskenpflicht aus medizinischen Gründen zu ermöglichen, bedürfe es für diesen Nachweis der Vorlage eines aktuellen ärztlichen Attestes, das den darin genannten Mindestanforderungen genügen müsse. Diese Glaubhaftmachung sei mit der Vorlage der jeweiligen ärztlichen Atteste nicht erfolgt. Den Antragstellern werde lediglich empfohlen, keine Maske zu tragen. Es fehle sowohl an einer konkreten Diagnose als auch an der Aussage, dass den Antragstellern das Tragen einer Mund-Nasen-Bedeckung nicht zumutbar ist. Es fehle zudem an der Benennung einer konkreten zu erwartenden gesundheitlichen Beeinträchtigung aufgrund des Tragens einer Mund-Nasen-Bedeckung im Unterricht sowie der Angabe, woraus diese im Einzelnen resultiere. Ein Rückschluss auf die in $\$ 3$ Abs. 6 CoronaVO genannten Vorerkrankungen sei folglich nicht möglich.

Soweit sich die ASt. unter Berufung auf den Schutz des Persönlichkeitsrechts und der DSGVO die Vorlage eines den Mindestanforderungen entsprechenden Attest verweigerten, ändere dies nichts daran, dass die Benennung einer Diagnose unabdingbar für die Nachvollziehbarkeit des Attests sei. Eine Befreiung vom Tragen der Mund-Nasen-Bedeckung stelle eine Ausnahme von der grundsätzlichen Regelung der $\$ ₫ 3$ Abs. 1, 13 Abs. 1 S. 6 CoronaVO dar, die dem Gesundheitsschutz der Allgemeinheit diene. Das Erfordernis, die Allgemeinheit vor einem erhöhten Ansteckungsrisiko zu schützen, würde durch die Befreiung von Personen, die nicht glaubhaft machen könnten, aus gesundheitlichen Gründen vom Tragen der Mund-Nasen-Bedeckung befreit zu sein, konterkariert. Daher müsse das allgemeine Persönlichkeitsrecht hinter dem hohen Schutzgut der Gesundheit der Allgemeinheit zurückstehen. [...]

Aus den Gründen: Der Antrag auf Gewährung vorläufigen Rechtsschutzes hat insgesamt keinen Erfolg. Er ist zulässig aber unbegründet.

Das VG kann nach $₫ 123$ Abs. 1 S. 2 VwGO auf Antrag eine einstweilige Anordnung zur Regelung eines vorläufigen Zustands in Bezug auf ein streitiges Rechtsverhältnis erlassen, wenn diese Regelung, um wesentliche Nachteile abzuwenden oder drohende Gewalt zu verhindern oder aus anderen Gründen erforderlich ist (sog. Regelungsanordnung). Voraussetzung dafür, dass das Gericht eine solche Regelungsanordnung erlassen kann, ist, dass der ASt. die Eilbedürftigkeit (den Anordnungsgrund) und sein subjektiv-öffentliches Recht (den Anordnungsanspruch) glaubhaft macht (\$123 Abs. 3 VwGO i. V. mit $\$ 920$ Abs. 2 ZPO).

Das einstweilige Rechtsschutzverfahren nach $\$ 123$ VwGO dient grundsätzlich nur der vorläufigen Regelung 
eines Rechtsverhältnisses; einem ASt. soll hier regelmäBig nicht bereits das gewährt werden, was er nur in einem Hauptsacheverfahren erreichen kann. Die von den Antragstellern begehrte Feststellung stellt sich als eine endgültige Vorwegnahme der Hauptsache dar. Wird - wie hier - die Hauptsache vorweggenommen, kann dem Eilantrag nach $\$ 123$ VwGO nur stattgegeben werden, wenn dies zur Gewährung effektiven Rechtsschutzes nach Art. 19 Abs. 4 GG schlechterdings unabweisbar ist. Dies setzt hohe Erfolgsaussichten, also eine weit überwiegende Wahrscheinlichkeit eines Erfolgs in der Hauptsache, sowie schwere und unzumutbare, nachträglich nicht mehr zu beseitigende Nachteile im Falle des Abwartens in der Hauptsache voraus (vgl. OVG Hamburg, Beschl. v. 6.7.2018 - 3 Bs 97/18 -, juris, Rdnr. 35).

Unter Berücksichtigung dieser Maßstäbe haben die ASt. nicht glaubhaft gemacht, einen Anspruch auf Feststellung, dass sie am Regelunterricht ohne das Tragen einer MundNasen-Bedeckung teilnehmen dürfen, sowie dass sie nicht verpflichtet sind mitzuteilen, welche konkret zu benennende gesundheitliche Beeinträchtigung auf Grund des Tragens der Mund-Nasen-Bedeckung im Unterricht alsbald zu erwarten ist und woraus diese im Einzelnen resultiert oder aber relevante Vorerkrankungen konkret zu benennen, zu haben.

Gemäß $₫ 13$ Abs. 1 S. 4 der Nds. Verordnung über Maßnahmen zur Eindämmung des Corona-Virus SARSCoV-2 (Nds. Corona-Verordnung) v. 30.10.2020 (im Folgenden CoronaVO) hat außerhalb von Unterrichts- und Arbeitsräumen jede Person eine Mund-Nasen-Bedeckung in von der Schule besonders gekennzeichneten Bereichen zu tragen, in denen aufgrund der örtlichen Gegebenheiten die Einhaltung des Abstandsgebots nach $\ 2$ Abs. 2 S. 1 CoronaVO zwischen Personen, die nicht derselben Gruppe i.S.d. Satzes 1 angehören, nicht gewährleistet werden kann. Nach S. 6 besteht die Verpflichtung zum Tragen einer Mund-Nasen-Bedeckung an einer Schule auch während des Unterrichts der Sekundarbereiche I und II wenn in Bezug auf das Gebiet des Landkreises oder der kreisfreien Stadt, in dem die Schule gelegen ist (Standort der Schule), die Zahl der Neuinfizierten im Verhältnis zur Bevölkerung 50 oder mehr Fälle je 100.000 Einwohnerinnen und Einwohner kumulativ in den letzten sieben Tagen beträgt (Nr. 1) für die Dauer der Überschreitung der in Nr. 1 genannten Zahl der Neuinfizierten.

Rechtsgrundlage für $\$ 13$ CoronaVO sind $\$ 32$ S. 1 und 2 IfSG i. V. mit $\$ 28$ Abs. 1 S. 1 IfSG i. V. mit $\$ 28$ a Abs. 1 IfSG. $\$ 28$ Abs. 1 S. 1 IfSG bestimmt: ,Werden Kranke, Krankheitsverdächtige, Ansteckungsverdächtige oder Ausscheider festgestellt oder ergibt sich, dass ein Verstorbener krank, krankheitsverdächtig oder Ausscheider war, so trifft die zuständige Behörde die notwendigen Schutzmaßnahmen, insbesondere die in den $\$ 28$ a Abs. 1 und in den $\$ \$ 29$ bis 31 genannten, soweit und solange es zur Verhinderung der Verbreitung übertragbarer Krankheiten erforderlich ist; sie kann insbesondere Personen verpflichten, den Ort, an dem sie sich befinden, nicht oder nur unter bestimmten Bedingungen zu verlassen oder von ihr bestimmte Orte oder öffentliche Orte nicht oder nur unter bestimmten Bedingungen zu betreten." Nach $\$ 28 \mathrm{a}$ Abs. 1 Nr. 2 IfSG kann insbesondere die Verpflichtung zum Tragen einer Mund-Nasen-Bedeckung (Maskenpflicht) notwendige Schutzmaßnahme i.S.d. $\$ 28$ Abs. 1 S. 1 und 2 zur Verhinderung der Verbreitung der Corona-Virus-Krankheit-2019 (COVID-19) für die Dauer der Feststellung einer epidemischen Lage von nationaler Tragweite nach $₫ 5$ Abs. 1 S. 1 durch den Deutschen Bundestag sein.

Die Verpflichtung zum Tragen einer Mund-NasenBedeckung stellt danach grundsätzlich eine zulässige Schutzmaßnahme dar (vgl. hierzu Nds. OVG, Beschl. v. 14. 8.2020 - 13 MN 300/20 -, juris, Rdnr. 12). Dies gilt auch für eine Verpflichtung, eine Mund-NasenBedeckung in der Schule, insbesondere auch während des Unterrichts zu tragen (vgl. Nordrhein-Westfälisches OVG, Beschl. v. 20.8.2020 - 13 B 1197/20.NE -, juris, 37; Bay. VGH, Beschl. v. 8. 9. 2020 - 20 NE 20.1999 -, juris). Die Verpflichtung ist auch verhältnismäßig. Das Robert-Koch-Institut fasst in seinem täglichen Lagebericht zur Coronavirus-Krankheit-2019 (COVID-19) (Stand 18.11.2020) zusammen, dass die hohen bundesweiten Fallzahlen zumeist durch diffuse Geschehen, mit zahlreichen Häufungen unter anderem in Haushalten, aber zunehmend auch in Gemeinschaftseinrichtungen verursacht werden. Da die Zahl der infizierten Personen derzeit in Deutschland sehr hoch sei, bedeute dies weiterhin eine hohe Zahl von täglichen Neuerkrankungen. Es sei weiterhin notwendig, ,dass sich die gesamte Bevölkerung für den Infektionsschutz engagiert, z. B. indem sie Abstands- und Hygieneregeln konsequent - auch im Freien - einhält, Innenräume lüftet und, wo geboten, eine Mund-Nasen-Bedeckung korrekt trägt. Menschenansammlungen - besonders in Innenräumen - sollten möglichst gemieden werden." [...] Das Robert-Koch-Institut hat darüber hinaus am 12.10.2020 Empfehlungen für Schulen herausgegeben, „Präventionsmaßnahmen in Schulen während der COVID-19-Pandemie“ [...] Darin heißt es unter anderem: „Die anerkannten Infektionsschutzmaßnahmen sind auch im Kindes- und Jugendalter wirksam, zumindest für ältere Kinder gut umsetzbar und ein wichtiger Baustein bei der Bewältigung der Pandemie.“ (S. 3). Eine Mund-Nasen-Bedeckung außerhalb des Unterrichts sowie auch im Klassenzimmer sollte bei einer Inzidenz von mehr als 50 pro 100.000 getragen werden (Tabelle S. 10). Die Maßnahme dient einem legitimen öffentlichen Zweck. Durch sie soll die Weiterverbreitung des SARS-CoV-2-Virus unter Schülern und Lehrern zumindest reduziert und hierdurch die Virusausbreitung insgesamt verringert werden. Dies wirkt einer unkontrollierten Infektionsausbreitung und der damit einhergehenden Gefahr einer Erkrankung einer Vielzahl von Menschen mit teilweise schwerwiegenden und tödlichen Krankheitsverläufen sowie einer Überforderung des Gesundheitssystems entgegen. Die Maßnahme ist auch geeignet, erforderlich und angemessen. Insoweit haben auch die ASt. nichts Gegenteiliges dargelegt.

Die vom Nds. Landesgesundheitsamt veröffentlichte 7-Tages-Inzidenz pro 100.000 Einwohner beträgt für den Landkreis A-Stadt am 19.11.2020, 9.00 Uhr 85,4 Fälle. Daher besteht für die ASt. grundsätzlich die Verpflichtung, einen Mund-Nasen-Schutz zu tragen.

Der AG. geht jedoch zutreffend davon aus, dass Schüler unter bestimmten Voraussetzungen von der Verpflichtung zum Tragen eines Mund-Nasen-Schutzes befreit sind.

Gemäß \$3 Abs. 6 CoronaVO sind Personen, für die aufgrund einer körperlichen, geistigen oder psychischen $\mathrm{Be}-$ einträchtigung oder einer Vorerkrankung, zum Beispiel einer schweren Herz- oder Lungenerkrankung, das Tragen einer Mund-Nasen-Bedeckung nicht zumutbar ist und die dies durch ein ärztliches Attest oder eine vergleichbare amtliche Bescheinigung glaubhaft machen können, von den Verpflichtungen nach den Absätzen 1, 2 und 5 ausgenommen. Diese Bestimmung nimmt nicht unmittelbar Bezug auf $\$ 13$ CoronaVO und nimmt daher die dort genannte Verpflichtung zum Tragen einer Mund-Nasen-Bedeckung nicht unmittelbar aus.

Gemäß $₫ 13$ Abs. 5 CoronaVO ist im Übrigen an allen Schulen der ,Nds. Rahmen-Hygieneplan Corona Schule“ v. 22.10.2020, veröffentlicht auf der Internetseite des Kultusministeriums [...], ergänzend zu den Hygieneplänen nach $\$ 36$ IfSG $\mathrm{zu}$ beachten. In diesem Rahmen-Hygieneplan heißt es unter Ziff. 6.4, 4. Abs. „Personen, für die 
aufgrund einer körperlichen, geistigen oder psychischen Beeinträchtigung oder einer Vorerkrankung, zum Beispiel einer schweren Herz- oder Lungenerkrankung, das Tragen einer MNB nicht zumutbar ist und die dies mit ärztlichem Attest glaubhaft machen können, sind von der Verpflichtung ausgenommen."

Im vorliegenden Fall kann letztlich dahinstehen, ob sich die Möglichkeit der Befreiung von der Maskenpflicht in der Schule unmittelbar aus $\$ 3$ Abs. 6 CoronaVO ergibt oder aus $\$ 13$ Abs. 5 CoronaVO i. V. mit dem „RahmenHygieneplan Corona Schule“v. 22. 10.2020. Denn der AG. ist erkennbar davon ausgegangen, dass eine Befreiung unter den genannten Voraussetzungen zu gewähren ist. Dies hat er mit Schreiben v. 2.11.2020 gegenüber den Erziehungsberechtigten der ASt. kundgetan.

Die ASt. haben nicht glaubhaft gemacht, dass ihnen das Tragen einer Mund-Nasen-Bedeckung aufgrund einer körperlichen, geistigen oder psychischen Beeinträchtigung oder einer Vorerkrankung, zum Beispiel einer schweren Herz- oder Lungenerkrankung nicht zumutbar ist. In den von ihnen vorgelegten Attesten heißt es lediglich, dass aus ärztlicher Sicht nicht empfohlen sei, die Maske zu tragen. Daraus lässt sich nicht auf eine Unzumutbarkeit des Tragens einer Mund-Nasen-Bedeckung schließen.

Zudem ist es rechtlich nicht zu beanstanden, dass die Rundverfügung Nr. 26/2020 zur Anwendung der Nds. Verordnung über Maßnahmen zur Eindämmung des Corona-Virus SARS-CoV-2 (Nds. Corona-Verordnung) v. 30.10.2020 unter Ziff. 1 Buchst. f vorsieht, dass soweit bei der Schule ein Befreiungstatbestand von der Pflicht zum Tragen einer Mund-Nasen-Bedeckung glaubhaft gemacht wird, sich aus einem aktuellen Attest oder einer aktuellen vergleichbaren amtlichen Bescheinigung nachvollziehbar ergeben muss, welche konkret zu benennende gesundheitliche Beeinträchtigung auf Grund des Tragens der Mund-Nasen-Bedeckung im Unterricht alsbald zu erwarten ist und woraus diese im Einzelnen resultiert; dass wenn relevante Vorerkrankungen vorliegen, diese konkret $\mathrm{zu}$ benennen sind und dass darüber hinaus im Regelfall erkennbar werden muss, auf welcher Grundlage die attestierende Ärztin oder der attestierende Arzt zu ihrer oder seiner Einschätzung gelangt ist. Die Befreiung vom Tragen einer Mund-Nasen-Bedeckung gewährt demjenigen, für den sie greift, einen rechtlichen Vorteil. In derartigen Konstellationen muss die Verwaltung beziehungsweise das Gericht, wie auch in anderen Rechtsgebieten, aufgrund konkreter und nachvollziehbarer Angaben in den ärztlichen Bescheinigungen in die Lage versetzt werden, das Vorliegen der jeweiligen Tatbestandsvoraussetzungen selbständig zu prüfen (Nordrhein-Westfälisches OVG, Beschl. v. 24.9. 2020 - 13 B 1368/20 -, juris, Rdnrn. 11, 12; Bay. VGH, Beschl. v. 26. 10.2020 - 20 CE 20.2185 -, juris, Rdnr. 19).

Soweit die ASt. geltend machen, dies verletze sie in ihrem allgemeinen Persönlichkeitsrecht aus Art. 2 Abs. 1 GG, ist dem entgegenzuhalten, dass vorliegend auch Grundrechtspositionen anderer Schülerinnen und Schüler sowie der Lehrkräfte und des übrigen Schulpersonals, nämlich das Recht auf Leben und Gesundheit aus Art. 2 Abs. 2 GG betroffen sind. Hierfür trägt die Schule eine herausgehobene Verantwortung (Bay. VGH, Beschl. v. 26.10.2020 20 CE 20.2185 -, juris, Rdnr. 19). Da die Maskenpflicht dazu dient, andere Personen vor einer Ansteckung mit dem neuartigen Coronavirus SARS-CoV-2 zu schützen und die Ausbreitungsgeschwindigkeit von COVID-19 in der Bevölkerung zu reduzieren, muss das allgemeine Persönlichkeitsrecht hierhinter zurückstehen.

Soweit sich die ASt. auf datenschutzrechtliche Erwägungen berufen, ist dem entgegenzuhalten, dass die Schule grundsätzlich berechtigt ist, die in einem solchen Attest enthaltenen personenbezogenen Daten der jewei- ligen Schülerin oder des jeweiligen Schülers zu verarbeiten. Die Rechtsgrundlage ergibt sich aus $\$ 31$ Abs. 1 Abs. 10 Nr. 1 Buchst. h NSchG. Nach $₫ 31$ Abs. 1 S. 1 NSchG dürfen Schulen personenbezogene Daten der Schülerinnen und Schüler und ihrer Erziehungsberechtigten verarbeiten, soweit dies zur Erfüllung der Fürsorgeaufgaben (Nr. 2) erforderlich ist. Gemäß \$31 Abs. 10 Nr. 1 Buchst. h NSchG dürfen von den besonderen Kategorien personenbezogener Daten i.S. d. Artikels 9 Abs. 1 der DS-GVO aufgrund der Regelungen in den Absätzen 1 bis 3 nur verarbeitet werden Gesundheitsdaten, soweit dies erforderlich ist, aus Gründen des öffentlichen Interesses im Bereich der öffentlichen Gesundheit und des Infektionsschutzes. Diese Voraussetzungen sind vorliegend erfüllt.

$[\ldots]$

\section{Anmerkung zu VG Braunschweig, Beschl. v. 19.11.2020 - 4 B 397/20}

\section{Benedikt Nethe}

Dem Beschluss des VG Braunschweig ist im Ergebnis vollumfänglich zuzustimmen. Die wesentlichen Problemfelder werden überwiegend in angemessener Tiefe abgehandelt.

Zentrale Themen der Entscheidung sind die Anforderungen an das beizubringende Attest nebst einschlägiger Rechtsgrundlagen sowie die gegeneinander im Wege der praktischen Konkordanz in Ausgleich zu bringenden Grundrechtspositionen. Ergänzend hat der Sachverhalt auch eine datenschutzrechtliche und strafrechtliche Dimension. Bemerkenswert ist die Entscheidung, weil sie konkret aufzeigt, dass und warum ein pauschales Attest für eine Befreiung von der Maskenpflicht nicht ausreichen kann. Vielmehr muss derjenige, der eine Befreiung erlangen möchte zwingend sensible Gesundheitsdaten offenbaren.

Gegenstand der Entscheidung war eine einstweilige Anordnung nach $\$ 123$ Abs. 1 S. 2 VwGO, da weder die Verordnung selbst ( $\$ 47$ Abs. 6 VwGO) noch ein belastender Verwaltungsakt ${ }^{1}$ angegriffen werden sollte ( $\$ 80 \mathrm{Abs} .5$ VwGO). Die Antragsteller begehrten die Feststellung, dass sie ohne das Tragen einer Mund-Nasen-Bedeckung am Regelunterricht teilnehmen dürfen. Ergänzend wurde auf Feststellung dahingehend geklagt, dass sie nicht verpflichtet seien, mitzuteilen, welche konkret zu benennende gesundheitliche Beeinträchtigung aufgrund des Tragens der Mund-Nasen-Bedeckung im Unterricht alsbald zu erwarten ist und woraus diese im Einzelnen resultiert oder aber relevante Vorerkrankungen konkret zu benennen. Die Schule war - soweit aus der Entscheidung ersichtlich - den entsprechenden Aufforderungen der Antragsteller schlicht nicht nachgekommen.

In den Gründen stellt das Gericht zunächst die Rechtsgrundlagen der Niedersächsischen Corona-Verordnung sowie der Maskenpflicht dar um sodann in der Verhältnismäßigkeitsprüfung den neuralgischen Punkt des Befreiungstatbestands zu prüfen.

Die Verpflichtung zum Tragen einer Mund-Nasen-Bedeckung während des Unterrichts in den Sekundarberei-

1) VG Düsseldorf, Beschl. v. 25.8.2020 - 18 L 1608/20.

Rechtsanwalt Benedikt Nethe,

CausaConcilio Koch \& Partner mbB Rechtsanwälte,

Bei den Gerichten - Deliusstraße 16, 24114 Kiel, Deutschland 